\title{
DEGRADAÇÃO FOTOELETROQUÍMICA DO CORANTE AZUL QR 19 EM SOLUÇÃO AQUOSA
}

\author{
Loraine C. V. Jacobs e Patricio G. Peralta-Zamora* \\ Departamento de Química, Universidade Federal do Paraná, CP 19081, 81531-990 Curitiba - PR, Brasil
}

Recebido em 20/5/11; aceito em 24/11/11; publicado na web em 23/1/12

\begin{abstract}
PHOTOELECTROCHEMICAL DEGRADATION OF REACTIVE BLUE 19 IN AQUEOUS SOLUTIONS. In this work the degradation capacity of a photo-electrocatalytic system was evaluated, mainly regarding the effect of the electrolyte solution on the degradation capacity toward a reactive textile dye. In the presence of $\mathrm{NaCl}$ the photo-electrochemical process shows high degradation efficiency, permitting almost total color removal in treatment of about $5 \mathrm{~min}$. In view of the low degradation efficiency observed for the photocatalytic process it is possible to assume that the high degradation efficiency of the process is a function of electrochemical generation of oxidant active chlorine species, which are subsequently transformed to higher oxidant radical forms.
\end{abstract}

Keywords: photo-electrocatalysis; dyes; electrolytes.

\section{INTRODUÇÃO}

O crescimento populacional e industrial que caracteriza as últimas décadas tem propiciado um significativo aumento na geração de resíduos urbanos e industriais, os quais, tratados ou dispostos de maneira inadequada, propiciam o surgimento de efeitos deletérios em diversos compartimentos ambientais.

Dentro do contexto da poluição de origem industrial, deve ser dado destaque aos processos de beneficiamento têxtil, particularmente aos processos de tingimento, os quais são responsáveis pela geração de grandes volumes de resíduos, contendo elevada carga orgânica e forte coloração. ${ }^{1}$ De maneira geral, estima-se que 1 a $15 \%$ da carga de corantes pode ser perdida durante operações de tingimento e lavagem, ${ }^{2}$ o que dificulta o tratamento e praticamente inviabiliza o reuso das águas residuárias. ${ }^{3}$

No meio industrial, o tratamento deste tipo de resíduos é realizados por rotinas convencionais, normalmente envolvendo processos de coagulação, adsorção e tratamento biológico, técnicas estas que apresentam inconvenientes derivados da elevada produção de lodos, do caráter não destrutivo e da usual resistência dos corantes frente a processos de natureza biológica, respectivamente. ${ }^{4}$

Em função das desvantagens associadas ao uso de processos convencionais de tratamento, a potencialidade dos processos oxidativos avançados tem sido investigada desde 1996, principalmente utilizando-se processos de fotocatálise heterogênea mediados por dióxido de titânio. ${ }^{5}$ Nestes estudos, a rápida degradação de corantes em solução aquosa tem sido demonstrada, principalmente utilizando-se fotocatalisador em suspensão. ${ }^{6-9}$

Mesmo apresentando elevada eficiência de degradação, poucos trabalhos relatam o uso do processo fotocatalítico em grande escala, basicamente em função de inconvenientes representados pela necessidade de fontes artificiais de radiação e de agentes sequestrantes de elétrons, bem como pela dificuldade de remoção do semicondutor, devido ao pequeno tamanho das partículas. ${ }^{10}$ Visando a minimização destes inconvenientes, novas alternativas vêm sendo propostas, com destaque para o uso de processos fotoeletroquímicos, os quais têm-se mostrado eficazes na degradação de poluentes orgânicos persistentes, em tempos de reação relativamente baixos. ${ }^{11,12}$ Neste processos, a imobilização do fotocatalisador na superfície de um eletrodo torna desnecessária a implementação de processos de separação do semicondutor,

*e-mail: zamora@.ufpr.br enquanto que a aplicação de um potencial anódico externo favorece o processo de separação de cargas e, consequentemente, maximiza a geração de radical hidroxila. ${ }^{12}$ Adicionalmente, estima-se que o uso de eletrodos do tipo DSA (Dimensionally Stable Anodes, por exemplo, $\mathrm{Ti} / \mathrm{Ru}_{0.3} \mathrm{Ti}_{0.7} \mathrm{O}_{2}$ ) viabiliza a geração de quantidades adicionais de radical hidroxila, em função da decomposição da água. ${ }^{13}$

Desta forma, a ocorrência de efeitos sinérgicos que melhoram significativamente a eficiência do processo de degradação é frequentemente relatada em estudos de degradação por processos fotoeletroquímicos. ${ }^{14}$

O presente trabalho teve como principal objetivo caracterizar o processo fotoeletroquímico frente à degradação do corante reativo azul QR 19, principalmente em relação ao efeito da natureza química do eletrólito utilizado. Embora estes aspectos sejam escassamente discutidos na literatura, antecedentes sugerem se tratar de uma característica que comanda os mecanismos de reação envolvidos no processo.

Até onde foi possível investigar, não existem artigos que relatem a degradação fotoeletroquímica do corante azul QR 19. Entretanto, vários trabalhos tratam da degradação de corantes têxteis por processos fotoeletroquímicos, utilizando ânodos de diversas naturezas. Utilizando um ânodo do tipo DSA, ${ }^{15}$ uma fonte de radiação ultravioleta de $250 \mathrm{~W}$ e sulfato de sódio como eletrólito, Catanho e colaboradores verificaram a remoção praticamente completa da cor de soluções aquosas contendo o corante vermelho reativo 198, em tratamentos da ordem de $90 \mathrm{~min}$. Por outro lado, mineralizações de apenas $40 \%$ foram verificadas em idêntico tempo de reação.

Recentemente, um interessante trabalho sobre degradação fotoeletroquímica de corantes dispersos foi publicado, utilizando-se um eletrodo de $\mathrm{TiO}_{2}$ imobilizado em uma placa de titânio. ${ }^{16}$ Neste estudo, a maior eficiência de degradação foi observada em tratamentos realizados na presença de cloreto de sódio, em função da geração de radical cloro, agente extremamente oxidante frente a corantes deste tipo.

\section{PARTE EXPERIMENTAL}

\section{Reagentes}

O corante reativo Azul QR 19 (CI 61200, Sigma) foi utilizado em solução aquosa em concentração de $50 \mathrm{mg} \mathrm{L}^{-1}$. Como eletrólitos foram utilizados $\mathrm{NaCl}$ e $\mathrm{Na}_{2} \mathrm{SO}_{4}$ (Vetec, grau analítico). 


\section{Tratamento fotoeletroquímico}

Todos os experimentos foram realizados em um reator de 750 $\mathrm{mL}$ de capacidade, equipado com camisa para refrigeração por água e sistema de agitação magnética. A radiação foi fornecida por uma lâmpada a vapor de mercúrio de $125 \mathrm{~W}$ de potência, inserida na parte central do reator com auxílio de um bulbo de quartzo. Os eletrodos utilizados possuem formato cilíndrico e foram inseridos de maneira concêntrica, mantendo uma distância de $1 \mathrm{~cm}$. Como cátodo foi utilizada uma tela de titânio de $97,5 \mathrm{~cm}^{2}$, enquanto que, como ânodo, uma placa de $\mathrm{Ti} / \mathrm{TiO}_{2} \mathrm{RuO}_{2}\left(\mathrm{DSA}^{\circledR}\right)$ com área de $138 \mathrm{~cm}^{2}$. A corrente foi fornecida por uma fonte externa (EMG 18134), controlada com o auxílio de um multímetro.

Para aplicação do processo fotoeletroquímico todos os componentes foram acionados simultaneamente, enquanto que nos estudos envolvendo processos isolados, apenas os componentes pertinentes. Isto é, fotólise (lâmpada ligada, na ausência de eletrodos), fotocatálise heterogênea (lâmpada ligada na presença de eletrodos, mas sem circulação de corrente) e eletrólise (na presença de eletrodos e circulação de corrente).

Neste reator, soluções aquosas do corante $(700 \mathrm{~mL}, \mathrm{pH}$ 6) foram adicionadas de eletrólito $\left(\mathrm{NaCl}\right.$ ou $\left.\mathrm{Na}_{2} \mathrm{SO}_{4}\right)$ e tratadas por tempos de até $120 \mathrm{~min}$. Alíquotas de $20 \mathrm{~mL}$ foram coletadas em intervalos regulares, sendo submetidas a controle analítico. Nos casos em que mais do que 5 amostras precisaram ser coletadas, o experimento foi dividido em 2 ou 3 rodadas, de maneira a não permitir que o nível da solução ficasse abaixo do nível da lâmpada ou dos eletrodos.

\section{Controle analítico}

A degradação fotoeletroquímica do corante reativo azul QR 19 foi avaliada por espectroscopia eletrônica (190 a $800 \mathrm{~nm}$ ), utilizando-se espectrofotômetro Shimadzu UV-2401 PC e cubetas de quartzo de $1 \mathrm{~cm}$ de caminho ótico.

A determinação de cloreto em solução foi realizada por método potenciométrico, utilizando-se eletrodo íon-seletivo Orion. Alíquotas de $10 \mathrm{~mL}$ de solução a ser analisada foram adicionadas de $10 \mathrm{~mL}$ de solução ajustadora de força iônica $\left(\mathrm{NaNO}_{3}, 0,1 \mathrm{~mol} \mathrm{~L}^{-1}\right)$ e medidas diretamente. A concentração de cloreto foi determinada por calibração externa, utilizando-se padrões de $\mathrm{NaCl}$ em concentração entre 0,0025 e $0,015 \mathrm{~mol} \mathrm{~L}^{-1}$.

O monitoramento da concentração de hipoclorito presente em solução foi realizado através de teste colorimétrico (Microquant 1.4879.0001, Merck $\left.^{\circledR}\right)$, fundamentado na reação de cloro, em meio levemente ácido, com N,N-Dietil p-fenilenodiamina (DPD), com formação de um composto que absorve fortemente em $512 \mathrm{~nm} .{ }^{17}$ A concentração de cloro foi determinada por calibração externa, utilizando-se padrões de hipoclorito de sódio em concentrações 0,001 a $0,010 \mathrm{~mol} \mathrm{~L}^{-1} \mathrm{e} 4 \times 10^{-5}$ a $4 \times 10^{-4} \mathrm{~mol} \mathrm{~L}^{-1}$.

\section{RESULTADOS E DISCUSSÃO}

\section{Degradação do corante reativo azul QR 19 na presença de sulfato de sódio}

Inicialmente, foi investigado o efeito de variáveis experimentais de relevância por planejamento fatorial de experimentos, utilizando-se a descoloração do corante como resposta analítica. Esse estudo envolveu as variáveis: $\mathrm{pH}$, concentração de eletrólito e densidade de corrente $\left(\mathrm{J}, \mathrm{mA} \mathrm{cm}^{-2}\right)$, cada uma das quais foi estudada em níveis definidos a partir de antecedentes descritos na literatura (Figura 1). Esta representação geométrica, que mostra valores de descoloração em tempos de reação de $40 \mathrm{~min}$, permite observar pouca influência das condições operacionais na capacidade de degradação apresentada pelo sistema. Exceção feita à densidade de corrente (J: 16), que mostra um efeito favorável e condizente com as informações encontradas na literatura especializada. ${ }^{18}$ Em função destes resultados, e levando-se em consideração o desvio médio calculado com base no experimento em triplicata $(0,3 \%)$, as condições do ponto central foram selecionadas para a realização das etapas subsequentes $\left(\mathrm{J}: 10 \mathrm{~mA} \mathrm{~cm}{ }^{-2}\right.$; $\mathrm{pH}: 6,0$; $\left.\mathrm{Na}_{2} \mathrm{SO}_{4}: 0,03 \mathrm{~mol} \mathrm{~L}^{-1}\right)$.

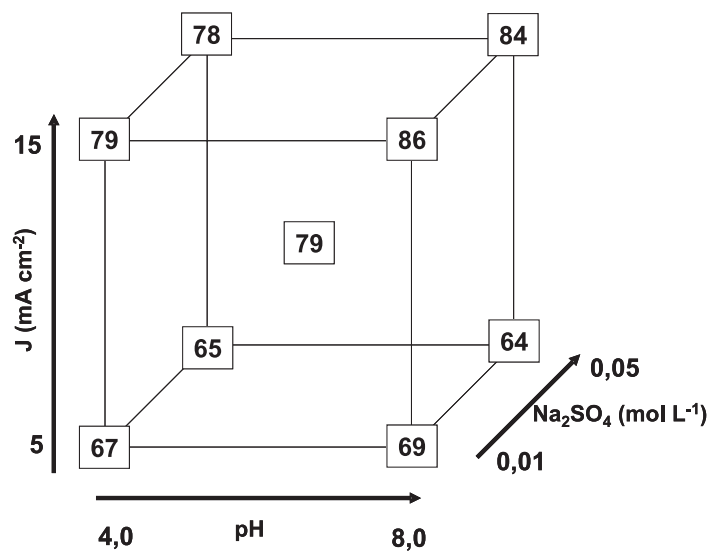

Figura 1. Representação geométrica do planejamento fatorial utilizado para otimização do processo fotoeletroquímico na presença de $\mathrm{Na}_{2} \mathrm{SO}_{4}$. (Efeitos principais: $\mathrm{pH}$ : 3; $\mathrm{J}: 16$; $\mathrm{Na}_{2} \mathrm{SO}_{4}:-2$; Desvio típico: 0,3 )

Para ilustrar o processo de degradação, apresenta-se na Figura 2 uma sequência de espectros coletados durante a degradação fotoeletroquímica do corante reativo azul QR 19, nas condições do ponto central. Estes resultados demonstram a degradação progressiva do grupo cromóforo, o que implica em descoloração praticamente completa em tempos de reação inferiores a 60 min. Os sinais registrados na região ultravioleta, tipicamente correspondentes a funções aromáticas, apresentam-se mais resistentes, permanecendo até nos maiores tempos de degradação praticados.

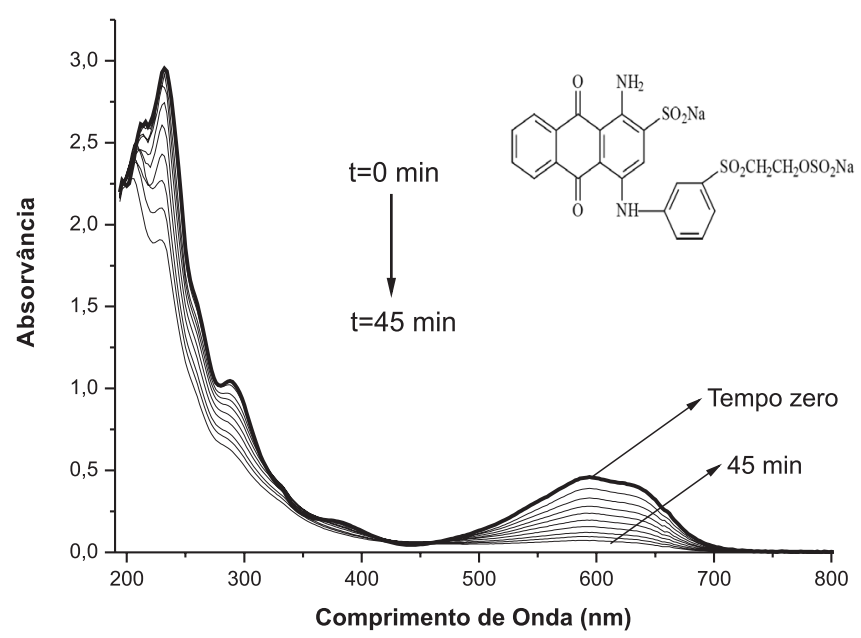

Figura 2. Sequência de espectros registrados durante a degradação fotoeletroquímica do corante reativo azul QR 19, na presença de sulfato de sódio ( J: $10 \mathrm{~mA} \mathrm{~cm} \mathrm{~cm}^{-2}$; $\mathrm{H}: 6,0 ; \mathrm{Na}_{2} \mathrm{SO}_{4}: 0,03 \mathrm{~mol} \mathrm{~L}^{-1}$ )

Normalmente, é aceito que a eficiência do sistema fotoeletroquímico se relaciona estreitamente com a densidade de corrente aplicada e com a concentração do eletrólito, ${ }^{18}$ na medida em que estes parâmetros influenciam fortemente a ocorrência de processos de 
natureza eletroquímica, os quais, por sua vez, favorecem o processo fotocatalítico. Em função da pouca influência observada neste estudo, é possível presumir uma baixa eficiência do processo fotocatalítico, principalmente em função da pequena quantidade de semicondutor imobilizada na superfície do eletrodo.

Em um processo fotoeletroquímico a degradação observada pode receber a contribuição de 4 processos concomitantes: fotólise (presença isolada de radiação), fotocatálise heterogênea (eletrodo contendo dióxido de titânio submetido à radiação), eletrólise (eletrodos submetidos a potencial externo) e processo fotoeletroquímico completo. Para avaliar cada uma destas contribuições, ensaios de degradação foram realizados nas condições do ponto central, observando-se os resultados apresentados na Figura 3. Estes resultados indicam que o processo de fotólise induz significativas mudanças na molécula de corante, o que permite descolorações da ordem de $20 \%$, em tratamentos de 60 min. Em idêntico tempo de reação, o processo de fotocatálise heterogênea permite descolorações da ordem de 35\%, o que se mostra relativamente baixo, principalmente levando-se em consideração o efeito isolado da radiação. Um comportamento similar é observado durante a aplicação de processos de eletrólise, com descolorações típicas de 30 a 35\%, em 60 min de reação.

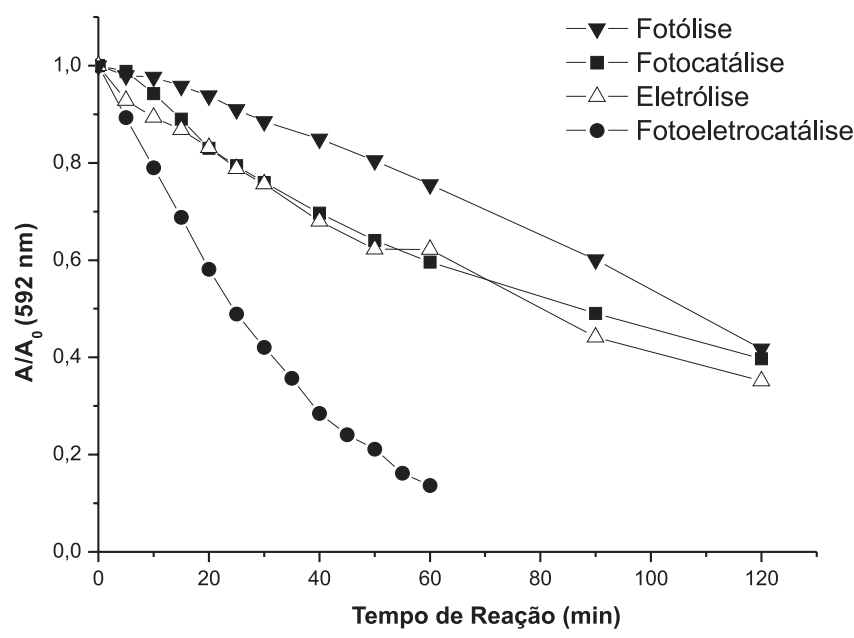

Figura 3. Contribuição dos processos isolados na degradação fotoeletroquímica do corante reativo azul QR 19 modelo na presença de sulfato de sódio ( $\mathrm{J}: 10 \mathrm{~mA} \mathrm{~cm} \mathrm{~cm}^{-2}$ pH: 6,0; $\mathrm{Na}_{2} \mathrm{SO}_{4}: 0,03 \mathrm{~mol} \mathrm{~L}^{-1}$ )

Um antecedente de relevância está representado pelo efeito sinérgico observado entre as componentes foto e eletroquímica do sistema. Isto é, enquanto a soma das taxas de descoloração permitidas pelos processos fotocatalíticos e eletrolíticos alcança valores da ordem de $75 \%$, o processo fotoeletrocatalítico permite descolorações substancialmente maiores, tipicamente da ordem de $90 \%$. Trata-se de uma observação que demonstra o efeito benéfico da associação de ambos os processos, usualmente relacionado com o favorecimento eletroquímico dos processos fotocatalíticos. ${ }^{19}$

\section{Degradação do corante reativo azul QR 19 na presença de cloreto de sódio}

Visando explorar de maneira mais aprofundada as reações fotoeletrocatalíticas, principalmente objetivando melhorar a eficiência do processo de degradação, um novo estudo foi realizado na presença de $\mathrm{NaCl}$. Como de costume, o efeito das principais variáveis operacionais foi investigado por um sistema de planejamento fatorial de experimentos, de acordo com o design apresentado na Figura 4.

A utilização de cloreto de sódio provocou significativos aumentos

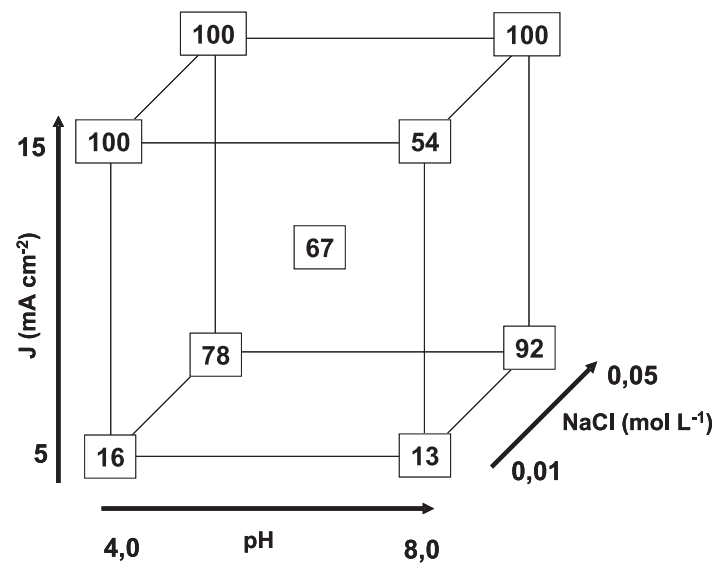

Figura 4. Representação geométrica do planejamento fatorial utilizado para otimização do processo fotoeletroquímico na presença de $\mathrm{NaCl}$. (Efeitos principais: pH: -9; J: 39; $\mathrm{NaCl}$ : 47; Desvio típico: $2 \%$ )

na velocidade da reação de degradação, o que permitiu a remoção praticamente completa da cor em tempos de reação inferiores a 5 min. Nas condições do ponto central, por exemplo, o monitoramento espectrofotométrico (resultados não apresentados) indicou que a cor é removida em uma grande extensão no primeiro minuto de reação, sendo praticamente imperceptível nas alíquotas coletadas a partir do segundo minuto.

Os resultados do planejamento, expressos na forma de percentual de descoloração em 1 min de reação (Figura 4), assim como os efeitos calculados, indicam que a eficiência do processo de degradação é significativamente favorecida pelo aumento da concentração do eletrólito e da densidade de corrente aplicada (efeitos principais de +47 e +39 pontos percentuais, respectivamente), o que é coerente com informações publicadas na literatura especializada. ${ }^{18}$ Por sua vez, o efeito do $\mathrm{pH}$ é negativo (efeito principal de -9 pontos percentuais), o que implica favorecimento do processo de degradação em menores valores de $\mathrm{pH}$.

Alguns estudos têm demonstrado a existência de uma competição entre os íons $\mathrm{HO}^{-}$e $\mathrm{Cl}^{-}$pela adsorção na superfície do fotocatalisador. De maneira geral, estima-se que a adsorção de $\mathrm{Cl}^{-}$é favorecida em meio ácido ( $\mathrm{pH}$ 1,0-4,5), o que redunda na formação de espécies radicalares de cloro $\left(\mathrm{Cl}^{*}\right.$ e $\left.\mathrm{Cl}_{2}{ }^{\circ}\right)$, de elevado potencial oxidante. ${ }^{16}$

$\mathrm{O}$ favorecimento do processo de degradação para maiores concentrações de eletrólito e maiores valores de corrente é coerente com a ocorrência de reações de degradação indiretas, mediadas por espécies de cloro ativo de elevado poder oxidante (ácido hipocloroso - $\mathrm{E}^{\mathrm{o}}=1,5 \mathrm{~V}$, íon hipoclorito $-\mathrm{E}^{\mathrm{o}}=0,89 \mathrm{~V}$ ), que surgem por oxidação eletroquímica do íon cloreto (Equações 1-3). ${ }^{20}$

$$
\begin{gathered}
2 \mathrm{Cl}^{-} \rightarrow \mathrm{Cl}_{2}+2 \mathrm{e}^{-} \\
\mathrm{Cl}_{2}+\mathrm{H}_{2} \mathrm{O} \rightarrow \mathrm{HClO}+\mathrm{H}^{+}+\mathrm{Cl}^{-} \\
\mathrm{HClO} \leftrightarrow \mathrm{H}^{+}+\mathrm{ClO}^{-}
\end{gathered}
$$

Assim como nos experimentos envolvendo $\mathrm{Na}_{2} \mathrm{SO}_{4}$, os processos envolvidos no sistema fotoeletrocatalítico foram analisados separadamente. Desta vez, o estudo não foi realizado nas condições experimentais que permitem a maior eficiência, em função das dificuldades de monitoramento encontradas nesta condição. Assim, os estudos foram realizados no $\mathrm{pH}$ natural da solução de corante (aproximadamente 6), utilizando-se a menor concentração de $\mathrm{NaCl}\left(0,01 \mathrm{~mol} \mathrm{~L}^{-1}\right)$ e a menor densidade de corrente $\left(5 \mathrm{~mA} \mathrm{~cm}^{-2}\right)$.

Os resultados (Figura 5) indicam que o processo de fotólise induz significativas mudanças na molécula de corante, o que permite descolorações da ordem de $30 \%$, em tratamentos de $60 \mathrm{~min}$. Em 
idêntico tempo de reação, o processo de fotocatálise heterogênea permite descolorações da ordem de $50 \%$, que se mostra relativamente baixo, principalmente levando-se em consideração o efeito isolado da radiação.

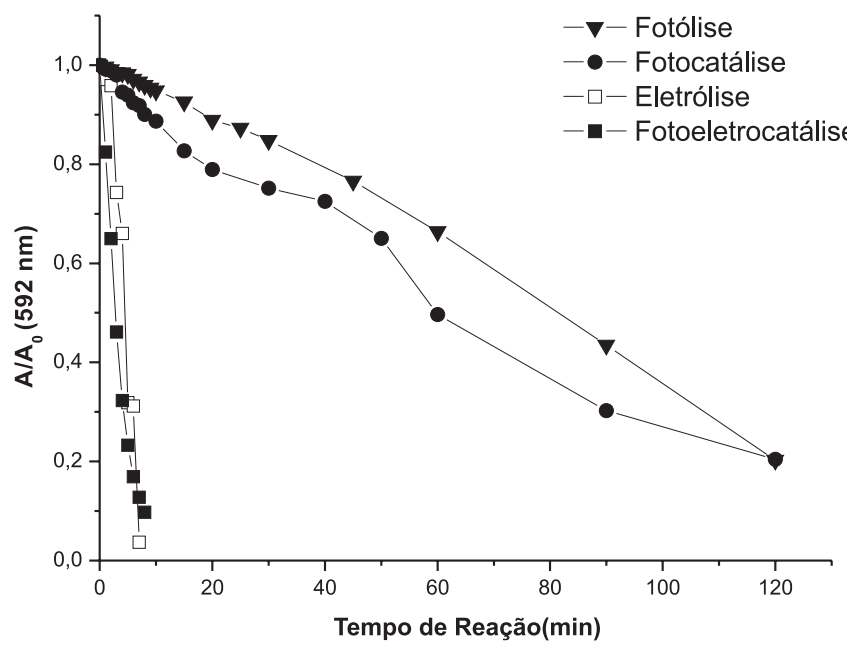

Figura 5. Monitoramento do teor de cloreto e hipoclorito durante a eletrólise de uma solução de cloreto de sódio 0,01 $\mathrm{mol} \mathrm{L}^{-1}$

Na presença de cloreto de sódio o processo eletroquímico se manifesta intensamente, superando largamente os processos concomitantes. Adicionalmente, a extrema semelhança observada no comportamento do corante frente aos processos eletroquímico e fotoeletroquímico sugere uma menor contribuição dos processos assistidos por radiação, o que sugere, novamente, um processo de degradação indireta, mediado por oxidantes clorados.

Para verificar esta hipótese, tanto o consumo de íon cloreto como o surgimento de cloro ativo foram monitorados. Para se evitar a interferência do corante e de subprodutos da sua degradação, estes experimentos foram realizados em solução aquosa contendo íon cloreto em concentração de 0,01 mol L-1. Os resultados (Figura 6) indicam um rápido consumo de íon cloreto e a manutenção de concentrações equivalentes a $50 \%$ da concentração inicial, até os maiores tempos ensaiados (90 min). Hipoclorito é produzido concomitantemente, alcançando concentrações de aproximadamente $3,2 \times 10^{-4} \mathrm{~mol} \mathrm{~L}^{-1}$ nos primeiros minutos de reação, o que corresponde a uma pequena

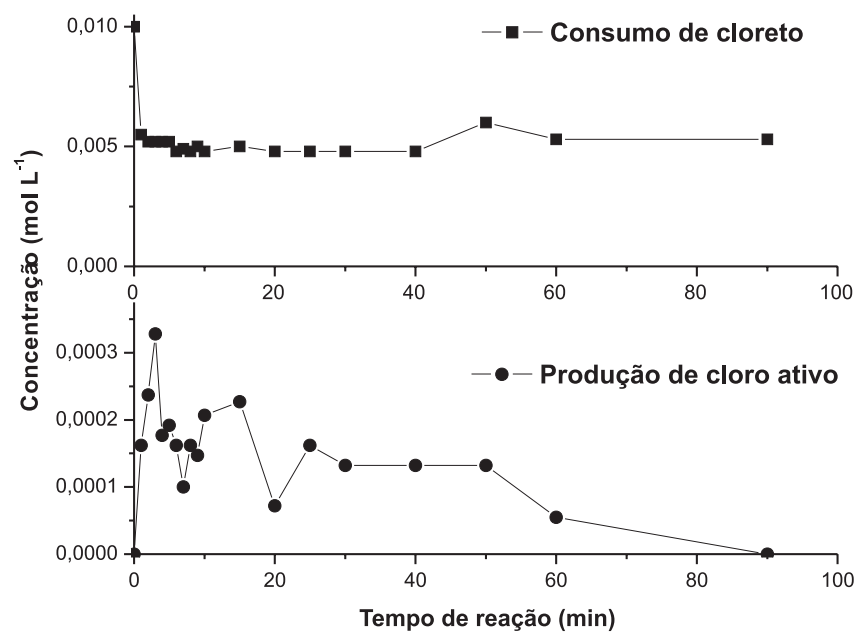

Figura 6. Degradação do corante reativo azul QR 19 por tratamento com hipoclorito de sódio $\left(3,2 \times 10^{-4} \mathrm{~mol} \mathrm{~L}^{-1}\right)$ na presença e ausência de radiação ultravioleta parcela do cloreto decomposto inicialmente. Este hipoclorito, mesmo na ausência do corante, tem sua concentração reduzida a aproximadamente zero no tempo final de monitoramento (90 $\mathrm{min}$ ).

Objetivando investigar a importância das reações mediadas por cloro, a solução de corante foi submetida à oxidação química, na presença de hipoclorito em concentração equivalente à gerada eletroquimicamente $\left(3,2 \times 10^{-4} \mathrm{~mol} \mathrm{~L}^{-1}\right)$. Nestas condições o grupo cromóforo da molécula de corante é efetivamente degradado, o que permite descolorações da ordem de $70 \%$ em 120 min de tratamento. Na presença de radiação ultravioleta o processo de degradação se mostra muito mais rápido, permitindo a remoção praticamente completa da cor em 4 min de tratamento (Figura 7).

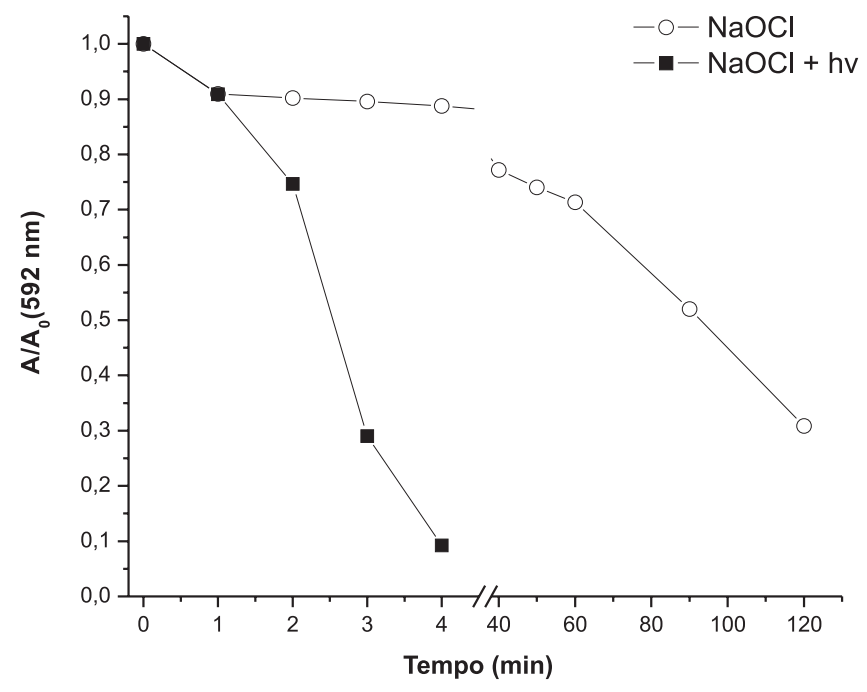

Figura 7. Degradação do corante reativo azul QR 19 por tratamento com hipoclorito de sódio $\left(3,2 \times 10^{-4} \mathrm{~mol} \mathrm{~L}^{-1}\right)$ na presença e ausência de radiação ultravioleta

O fato da reação se dar de maneira mais rápida no sistema assistido por radiação envolve, necessariamente, a ocorrência de reações fotoquímicas de relevância. O efeito favorável da radiação foi recentemente observado em estudos de degradação de amônia por processos eletroquímicos envolvendo o uso de cloreto de sódio. ${ }^{20}$ Neste estudo, a maior eficiência dos sistemas assistidos por radiação $(200-400 \mathrm{~nm})$ é atribuída à formação de radical cloro e radical hidroxila (Equação 4), espécies que, em função do seu elevado poder oxidante, podem promover a abstração de hidrogênio de hidrocarbonetos, desencadeando reações que aceleram o processo de degradação (Equação 5).

$$
\begin{gathered}
\mathrm{HOCl}+\mathrm{h} v \rightarrow{ }^{\bullet} \mathrm{OH}+{ }^{\cdot} \mathrm{Cl} \\
{ }^{\cdot} \mathrm{Cl}+\mathrm{HR} \rightarrow \mathrm{HCl}+\mathrm{R}^{\cdot}
\end{gathered}
$$

Com base nestes argumentos, é possível assumir que a elevada capacidade de degradação do processo fotoeletroquímico, quando aplicado na presença de eletrodos do tipo DSA e de eletrólitos ativos como $\mathrm{NaCl}$, é devida a reações eletroquímicas indiretas mediadas por espécies ativas de cloro, assistidas por processos de conversão fotoquímica que levam à formação de espécies de maior poder oxidante, como radical cloro e hidroxila. De acordo com antecedentes reportados por Osugi e colaboradores, ${ }^{16}$ a capacidade de mineralização dos processos fotoeletroquímicos mediados pela geração de formas ativas de cloro é marcadamente superior à capacidade demonstrada pela cloração química, basicamente em função da radiação permitir a formação de espécies radicalares de cloro. Nestas condições, foi observada mineralização praticamente completa no tratamento de soluções aquosas do corante disperso azul 291. 


\section{CONCLUSÕES}

Em função dos resultados observados é possível concluir que, quando na presença de sulfato de sódio, o processo fotoeletroquímico se processa de forma relativamente lenta, permitindo a remoção de cerca de $90 \%$ da cor em tempos de reação de 60 min. Mesmo assim, é possível observar um efeito sinérgico entre processos fotocatalíticos e eletroquímicos, o que faz com que a eficiência do processo combinado seja maior que a soma das contribuições de cada processo isolado.

$\mathrm{Na}$ presença de cloreto de sódio, o processo fotoeletroquímico mostrou uma elevada eficiência de degradação, o que permitiu a completa degradação do corante reativo azul QR 19 em tratamentos da ordem de 5 min. Em função da relativamente baixa eficiência de degradação observada no processo fotocatalítico é possível assumir que, nas condições em que este trabalho foi realizado, a elevada capacidade de degradação do processo fotoeletroquímico seja uma função da geração eletroquímica de espécies ativas de cloro (como hipoclorito) e da sua conversão fotoquímica em espécies radicalares de maior poder oxidante (como radicais cloro e hidroxila).

\section{REFERÊNCIAS}

1. Wang, K.; Chen, H.; Huang, L.; Su, Y.; Chang, S.; Chemosphere 2008, 72, 299.

2. Neelavannan, G. G.; Revathi, M.; Basha, C. A.; J. Hazard. Mater. 2007, 149, 371.

3. Constapel, M.; Schellenträger, M.; Marzinkowski, J. M.; Gäb, S.; Water Res. 2009, 43, 733.
4. Mahmoodi, N. M.; Arami, M.; J. Photochem. Photobiol., B 2009, 94, 20.

5. Fujishima, A.; Zhang, X.; Tryk, D. A.; Surf. Sci. Rep. 2008, 63, 515.

6. Konstantinou, I. K.; Albanis, T. A.; Appl. Catal., B 2004, 49, 1.

7. Aarthi, T.; Narahari, P.; Madras, G.; J. Hazard. Mater. 2007, 149, 725.

8. Gupta, V. K.; Jain, R.; Nayak, A.; Agarwal, S.; Shrivastava, M.; Mater. Sci. Eng., C. 2011, 31, 1062.

9. Asiri, A. M.; Al-Amoudi, M. S.; Al-Talhi, T. A.; Al-Talhi, A. D.; J. Saudi Chem. Soc. 2011, 15, 121.

10. Zlamal, M.; Macak, J. M.; Schmuki, P.; Krysa, J.; Electrochem. Commun. 2007, 9, 2822

11. Hou, Y.; Qu, J.; Zhao, X.; Lei, P.; Wan, D.; Huang, C. P.; Sci. Total Environ. 2009, 407, 2431.

12. Li, G.; Qu, J.; Zhang, X.; Ge, J.; Water Res. 2006, 40, 213.

13. Malpass, G. R. P.; Miwa, D. W.; Miwa, A. C. P.; Machado, S. A. S.; Motheo, A. J.; J. Hazard. Mater. 2009, 167, 224.

14. Quan, X.; Chen, S.; Su, J.; Chen, J.; Chen, G.; Sep. Purif. Technol. 2004, 34,73 .

15. Catanho, M.; Malpass, G. R. P.; Motheo, A. J.; Appl. Catal., B 2006, 62, 193.

16. Osugi, M. E.; Rajeshwar, K.; Ferraz, E. R. A.; de Oliveira, D. P.; Araújo, A. R.; Zanoni, M. V. B.; Electrochim. Acta 2009, 54, 2086.

17. APHA; Standard methods for the examinarion of water and wastewater; $19^{\text {th }}$ ed., WPCF: New York, 1995, 4500-Cl G, 4.

18. Pelegrini, R.; Zamora, P. P.; Appl. Catal., B 1999, 47, 219.

19. Bertazzoli, R.; Pelegrini, R.; Quim. Nova 2002, 25, 477.

20. Xiao, S.; Qu, J.; Zhao, X.; Liu, H.; Wan, D.; Water Res. 2009, 43, 1432. 